\title{
Metodologia Baseada em Assinatura Espectral Normalizada para Classificação Evolutiva de Imagens
}

\author{
Felipe P. Costa ${ }^{*}$ Selmo E. R. Junior ${ }^{* *}$ Luis M. M. Torres ${ }^{* * *}$ \\ * Instituto Federal de Educação, Ciência e Tecnologia do Maranhão / \\ Campus Imperatriz (e-mail: felipe.costa@acad.ifma.edu.br). \\ ** Instituto Federal de Educação, Ciência e Tecnologia do Maranhão / \\ Campus Imperatriz(e-mail: selmo.junior@ifma.edu.br). \\ *** Instituto Federal de Educação, Ciência e Tecnologia do Maranhão / \\ Campus Imperatriz (e-mail: luis.torres@ifma.edu.br).
}

\begin{abstract}
The present article proposes a methodology of image classification in an evolving way. For that is necessary to obtain the edge through classical methods of image processing as the contour detector. After obtaining the points belonging to the edge of an object it is calculated the distance from point to point, in order to generate a complex network, responsible for the description of the image and generation of a comparable and robust signature against distortion, change of scale and rotation . This signature is the input of the evolutionary algorithm responsible for grouping the images and able to fit the data set through the creation or changing of new sets while gather information. The resulting cluster proved to be efficient by correctly classifying $92 \%$ of the images of a sub-set of database.

Resumo: O presente artigo propõe uma metodologia de classificação de imagens de forma evolutiva. Para isso é necessária a obtenção da borda através de métodos clássicos de processamento de imagem, como o detector de contorno. Após a obtenção dos pontos pertencentes a borda de um objeto é calculada a distancia de ponto a ponto, a fim de gerar uma rede complexa responsável pela descrição da imagem e geração de uma assinatura comparável robusta frente a distorção, mudança de escala e rotação. Essa assinatura é a entrada para o algoritmo evolutivo, responsável por agrupar as imagens, com a capacidade de se adequar ao conjunto de dados através da criação ou alteração de novos grupos enquanto recebe informações. O agrupamento resultante se mostrou eficiente classificando corretamente $92 \%$ das imagens de um sub-conjunto de database pré-estabelecido.
\end{abstract}

Keywords: Signature Generation, Intelligent Image Processing, Pattern Recognition, Evolving Systems, computer vision.

Palavras-chaves: Geração de Assinatura, Processamento de Imagens Inteligente, Reconhecimento de Padrões, Sistemas Evolutivos, Visão Computacional.

\section{INTRODUÇÃO}

Segundo Jha et al. (2015), a indústria tem lidado com contínuas alterações e inovações, tanto na variedade de produtos quanto na sua confecção de maneira inteligente. A busca pela utilização de tecnologias que aumentem a velocidade e repetibilidade dos processos é cada vez maior e, segundo Thiago S et al. (2016), os sistemas de visão computacional tem se tornado paulatinamente estimados atingindo diferentes áreas e aplicações.

Dentre essas aplicações, além da indústria de manufatura, conforme trabalhada por Henrique R. Z. da Silva (2018) em seu trabalho de identificação de defeitos na linha de produção de frangos, podem ser citadas: a área de diagnóstico por imagem, como pode ser visto em Zago et al. (2016) que se utiliza da transformada de Houg para

\footnotetext{
* Fomentado pela Fundação de Amparo à Pesquisa e Desenvolvimento Científico do Maranhão - Fapema
}

identificação de Glaucoma a partir de fotos das retinas dos pacientes. As robóticas móvel e fixa exploradas por Corke (2017), que evidencia a ligação entre a visão e o controle do posicionamento dos autômatos. E o gerenciamento urbano no qual Lopes et al. (2015) se utiliza do Google Street View para obtenção de informações, a cerca dos postes que compõe a rede elétrica, através dos Descritores Elípticos de Fourier.

Facilmente observa-se que, mesmo em campos de pesquisa divergentes, há uma ampla utilização de descritores clássicos de forma e Redes Neurais Artificiais (RNA) como maneira de classificação, porém, enquanto a primeira possui dependências quanto a conformidade dos objetos a serem distinguidos, a segunda gera a necessidade de uma fase de treinamento demorada e um conjunto de dados com volume considerável diversas vezes indisponível.

Esta metodologia propõe uma alternativa ao treinamento das RNA, considerando uma abordagem evolutiva que 
atualize as classes existentes ou gere novas classes com base nos parâmetros da assinatura de imagem. Utilizando para isso uma combinação entre técnicas de visão e inteligência computacionais, como a extração de bordas de uma imagem, geração de uma assinatura comparável e, através de um agrupamento evolutivo on-line, a criação autônoma de classes de maneira inteligente.

Este trabalho apresenta a utilização de algoritmos evolutivos para a solução destes problemas, além de trabalhar de maneira a obter uma assinatura, que resulta em um menor custo computacional para a análise da forma em questão.

O artigo está segmentado em outras três sessões, sendo elas, a metodologia, os resultados e as conclusões. Na metodologia se concentra o cerne do trabalho onde são detalhadas as quatro principais etapas da classificação proposta. Em resultados é mostrado o desempenho da metodologia perante um conjunto de dados previamente estabelecido. Em conclusões há o encerramento do artigo e considerações finais.

\section{METODOLOGIA}

A metodologia de classificação proposta neste artigo é dividida em quatro módulos principais, conforme a figura 1, e que serão explanados nos tópicos a seguir, que são:

(i) Obtenção da Imagem:

(a) Conexão com a câmera ou banco de dados;

(b) Redimensionamento inicial da imagem;

(ii) Obtenção da Borda:

(a) Quantização;

(b) Aplicação de blur Gaussiano;

(c) Limiarização da imagem;

(d) Detector de bordas;

(iii) Obtenção da Assinatura:

(a) Geração da matriz de pesos;

(b) Obtenção da matriz adjacência;

(c) Obtenção dos auto-valores significantes;

(d) Normalização;

(iv) Classificação Evolutiva:

(a) Evolving Vector Quantization;

\subsection{Obtenção da Imagem}

A obtenção da imagem pode ser feita através de dois principais meios: Uma câmera ligada ao computador, ou um banco de dados previamente selecionado. No caso, para fins de comparação foi escolhida a utilização de sub-classes da database MPEG7 CE Shape 1 Part B geradas por Bicego et al. (2004). Em ambos os casos a imagem obtida pode ser representada como uma matriz tridimensional $I_{R G B}(3, N 1, N 2)$ no espaço de cores RGB, sendo $N 1$ e $N 2$, respectivamente, a largura e altura (em pixels) da imagem. Os valores de cada célula da matriz podem variar entre 0 e 255, para cada canal de cor (primeiro índice da matriz).

Nesta primeira etapa, está incluso o processo de redimensionamento, de forma que a imagem original se adéque à estrutura preparada para o processamento, assim, para uma maior eficiência foi escolhido o redimensionamento da imagem para o formato de $380 \times 420$, resultando em um total de 478.800 células, para a representação de cores no padrão RGB. Como afirma Corke (2017), essa redução é

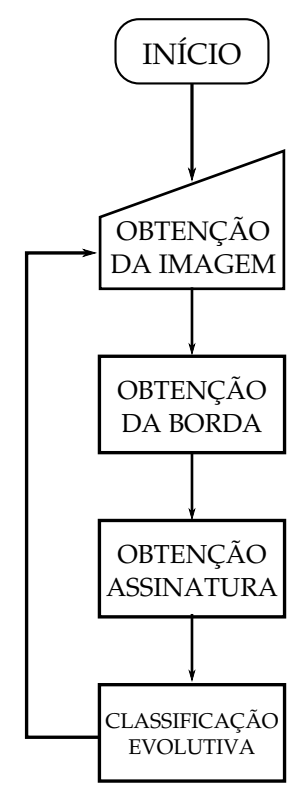

Figura 1. Fluxograma da Metodologia

almejada dado que o tempo e a memória requeridos para o processamento de uma imagem é proporcional as suas dimensões.

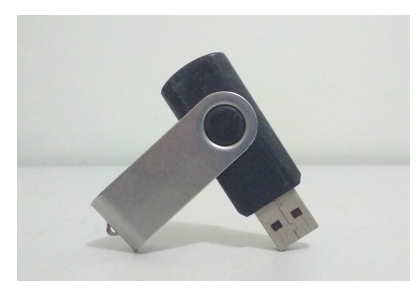

(a) Imagem Original

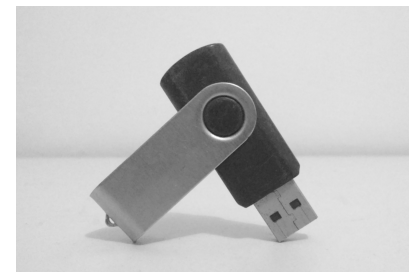

(c) Desfoque Gaussiano

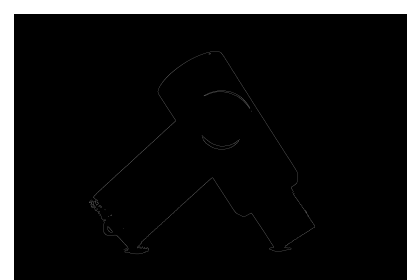

(e) Detector de Bordas

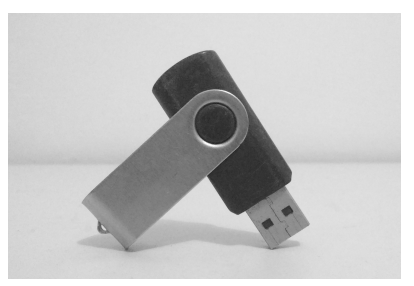

(b) Imagem Quantizada

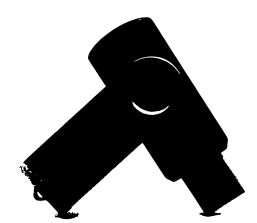

(d) Limiarização

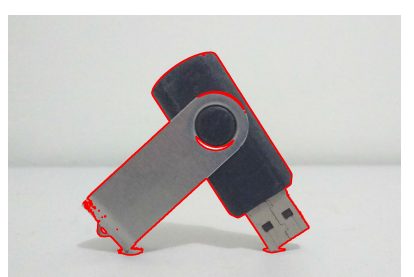

(f) Imagem com borda
Figura 2. Etapas para obtenção de borda

\subsection{Obtenção da Borda}

Visto que a matriz de representação da imagem, apresentada anteriormente, possui uma quantidade significativa de dados, diversas técnicas são aplicadas à imagem a fim de representá-la de outras maneiras. As imagens obtidas na etapa anterior são representações em duas dimensões da realidade em um determinado instante. E segundo Erpen 
(2004), neste caso existem dois tipos básicos de similaridades comparáveis de forma, as de região e as de contorno.

O presente trabalho se utiliza de descritores de contorno, já que estes apresentam, em geral, uma maior robustez à deformações plásticas na imagem e a distorções causadas pela movimentação ou redimensionamento da imagem. Este contorno é intuitivamente definido como um conjunto de pixels que fazem fronteira a regiões diferentes na imagem (Gonzalez and Woods, 2000).

Quantização Visto que se trata de um módulo de detecção de bordas, a informação acerca da cor pode ser suprimida através de uma transformação. De acordo com Zago et al. (2016), dá-se o nome de quantização para o processo de adaptação do padrão de cores para outro desejado.

$$
\begin{gathered}
I_{C i n z a}=\left[\begin{array}{cccc}
i_{c}(1,1) & i_{c}(1,2) & \cdots & i_{c}(1, j) \\
i_{c}(2,1) & i_{c}(2,2) & \cdots & i_{c}(2, j) \\
\vdots & \vdots & \ddots & \vdots \\
i_{c}(i, 1) & i_{c}(i, 2) & \cdots & i_{c}(i, j)
\end{array}\right] \\
i_{c}(i, j)=\frac{\sum_{k=1}^{3} I_{R G B}(k, i, j)}{3}
\end{gathered}
$$

A Equação (1a) apresenta a matriz $I_{\text {Cinza }}$ que descreve a imagem em escala de cinza onde $i_{c}$ são seus elementos que indicam que a transformação foi realizada através da média aritmética entre os $k$ canais da imagem original $I_{R G B}$.

Ainda segundo Erpen (2004) as regiões das quais se quer identificar as bordas, habitualmente, se mantém distinguíveis através dos níveis de cinza, obtidos através da quantização, ou ainda através das informações de apenas um de seus canais de cor.

O que de fato pode ser observado na transformação entre as figuras 2(a) e 2(b), as regiões presentes na imagem permanecem distinguíveis. Assim obtêm-se uma matriz de $I_{\text {Cinza }}$ com a mesma faixa de valores porém para 159.600 pixels.

Aplicação de Blur Gaussiano O desfoque das imagens, como visto na figura $2(\mathrm{c})$, é realizado para redução de eventuais ruídos gerados na transmissão da imagem ou ainda para remoção de detalhes que possam vir atrapalhar a identificação das bordas (Gonzalez and Woods, 2000). A nova matriz de representação é indicada em (2a) enquanto a operação utilizada para o desfoque é descrita em (2b).

$$
\begin{array}{r}
I_{\text {Blur }}=\left[\begin{array}{cccc}
i_{b}(1,1) & i_{b}(1,2) & \cdots & i_{b}(1, j) \\
i_{b}(2,1) & i_{b}(2,2) & \cdots & i_{b}(2, j) \\
\vdots & \vdots & \ddots & \vdots \\
i_{b}(i, 1) & i_{b}(i, 2) & \cdots & i_{b}(i, j)
\end{array}\right] \\
i_{b}(i, j)=\sum_{k=1}^{5} \sum_{l=1}^{5} I_{\text {Cinza }}(i+k, j+l) \star K(k, l)
\end{array}
$$

A imagem desfocada é representada pela matriz $I_{B l u r}$ e seus elementos $i_{b}$, que por sua vez, são o resultado de uma operação de convolução, denotada por $\star$, entre um determinado $K$ que é chamado Kernel, definido, para esta aplicação, através de (3a):

$$
\begin{aligned}
& K=\left[\begin{array}{cccc}
k(1,1) & k(1,2) & \cdots & k(1,5) \\
k(2,1) & k(2,2) & \cdots & k(2,5) \\
\vdots & \vdots & \ddots & \vdots \\
k(5,1) & k(5,2) & \cdots & k,(5,5)
\end{array}\right] \\
& k(i, j)=\frac{e^{-\left(\frac{(i-3)^{2}+(j-3)^{2}}{98}\right)}}{98 \pi}
\end{aligned}
$$

O Kernel utilizado possui a dimensão de $5 \times 5$, que é o valor mais comum na utilização desse filtro, visto que maiores dimensões requerem maior tempo de computação. Cada um dos seus elementos $k$ segue uma distribuição normal, que caracteriza a matriz, com a média centrada no elemento $\mathrm{k}(3,3)$.

Limiarização da Imagem A imagem binária contém regiões de intensidade constante, que é uma característica atrativa a essa aplicação, pois, segundo Gonzalez and Woods (2000), possibilita uma representação mais eficiente das bordas de um determinado objeto. A figura 2(d) apresenta o resultado dessa operação.

A técnica utilizada para a limiarização foi a proposta por Otsu (1979) e se baseia em manipulações dos valores obtidos através do histograma. É considerada uma boa prática utilizar esta técnica após a suavização, garantindo menores erros. O histograma referente à figura 2(c) pode ser visto na figura 3 (Gonzalez, 2010).

Sendo $p_{i}$ cada componente do histograma, a soma $P(k)$ é definida conforme (4a) para cada $k(0: K)$ limiar do histograma. De mesmo modo a média acumulada $m(k)$ é definida através de $(4 b)$.

$$
\begin{gathered}
P(k)=\sum_{i=0}^{k} p_{i} \\
m(k)=\sum_{i=0}^{k} i p_{i}
\end{gathered}
$$

A partir dos valores calculados é obtida a variância entre classes $\sigma^{2}(k)$ de acordo com (5a). O valor do limiar $k^{*}$ desejado é aquele que a maximiza.

$$
\begin{gathered}
\sigma^{2}(k)=\frac{[m(K) P(k)-m(k)]^{2}}{P(k)[i-P(k)]} \\
k^{*}=\underset{k}{\arg \max } \sigma^{2}(k)
\end{gathered}
$$

A figura 2(d) é o resultado da classificação de cada pixel da matriz 2 a através de (6a). Onde cada pixel é avaliado e convertido para um valor binário. 


$$
\begin{gathered}
I_{l}=\left[\begin{array}{cccc}
i_{l}(1,1) & i_{l}(1,2) & \cdots & i_{l}(1, j) \\
i_{l}(2,1) & i_{l}(2,2) & \cdots & i_{l}(2, j) \\
\vdots & \vdots & \ddots & \vdots \\
i_{l}(i, 1) & i_{l}(i, 2) & \cdots & i_{l}(i, j)
\end{array}\right] \\
i_{l}(i, j)=\left\{\begin{array}{lr}
0, & \text { se } i_{b}(i, j)>k^{*} \\
1, & \text { em outros casos }
\end{array}\right.
\end{gathered}
$$

Com $I_{l}$ sendo a matriz da imagem limiarizada e seus elementos $i_{l}$ definidos através de uma comparação entre os valores de $I_{B l u r}$ e $k^{*}$ obtido através de $(5 \mathrm{~b})$.

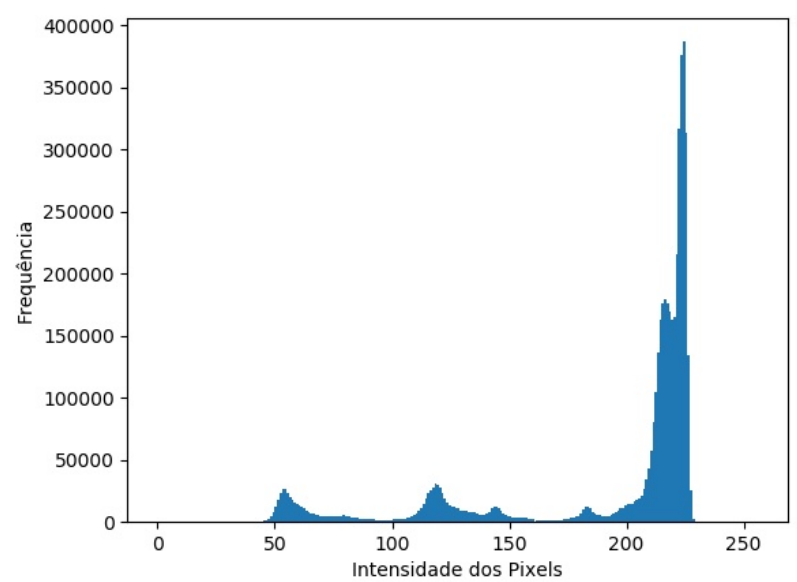

Figura 3. Histograma referente a figura 2(c)

Detector de Pontos da Borda Visto que a matriz obtida anteriormente é composta apenas de valores binários, seus contornos podem ser classificados como uma borda em degraus, onde a transição entre os níveis de intensidade das regiões possui tamanho unitário(Gonzalez, 2010). Assim, a verificação das bordas é calculada de maneira simples, ao analisar se esta variação existe em torno de cada ponto no qual $I_{l}=1$.

O resultado desse método pode ser observado na figura 2(e), onde apenas a borda é apresentada, e em 2(f) onde a borda é apresentada sobrepondo a imagem original.

\subsection{Obtenção de Assinatura}

Uma assinatura é um conjunto de características que descrevem uma determinada imagem. Esse artigo usa o Módulo da Assinatura Espectral Normalizada $\tilde{\xi}$ mod, proposta em Bof and Bof (2016). Segundo o autor citado, este descritor se baseia nas informações obtidas a partir do espectro da dinâmica da rede complexa associada à forma na qual se deseja caracterizar, para isso essa dinâmica é representada por um conjunto de matrizes de adjacência e culmina na geração de um vetor de assinatura.

Matriz de Pesos Inicialmente deseja-se descrever a borda em termos de rede complexa, para isso é calculada uma matriz de pesos, conforme (7a), onde cada um de seus elementos (7b) corresponde a distância euclidiana entre dois pontos do contorno, como visto a seguir:

$$
\begin{gathered}
W=\left[\begin{array}{cccc}
w_{1,1} & w_{1,2} & \cdots & w_{1, N} \\
w_{2,1} & w_{2,2} & \cdots & w_{2, N} \\
\vdots & \vdots & \ddots & \vdots \\
w_{N, 1} & w_{N, 2} & \cdots & w_{N, N}
\end{array}\right] \\
w_{i, j}\left(p_{i}, p_{j}\right)=\sqrt{\left(x_{i}-x_{j}\right)^{2}+\left(y_{i}-y_{j}\right)^{2}}
\end{gathered}
$$

Onde $p_{n}$ é um ponto $n$ do contorno, e $x_{n}$ e $y_{n}$ são suas coordenadas. Antes de prosseguir para a próxima etapa é necessário realizar a normalização da matriz em relação a maior distância. Segundo Bof and Bof (2016) essa operação tem a finalidade de relativizar os efeitos de suas magnitudes reduzindo consideravelmente a influência dos efeitos de escala. Essa normalização é feita através de (8) onde $\max ()$ é uma função que retorna o maior valor de seu argumento:

$$
\tilde{w}_{i, j}=\frac{w_{i, j}}{\max (W)}
$$

Matriz adjacência A geração da dinâmica da rede complexa é feita de maneira artificial através de uma matriz adjacência formada pelos pontos do contorno e as distâncias entre eles. Esta indica o valor 1 caso os pontos estejam ligados e a distância entre eles esteja entre $t$ e $t+0,1$, para $t=0,1$ a 0,9 no passo de 0,1 .

$$
m_{(i, j)_{t}}=\left\{\begin{array}{lc}
1, & \text { se } t \leq \tilde{w}_{i, j} \leq t+0,1 \\
0, & \text { em outros casos }
\end{array}\right.
$$

Autovalores e Normalização A assinatura é formada pelos 3 menores e maiores autovalores $(\lambda)$ a cada $t$ de $m_{(i, j)_{t}}$, conforme apresentado em (10a). Assim Bof and Bof (2016) descreve que elimina-se problemas oriundos da variação do número de autovalores em função do número de vértices que ocorre ao comparar formas com números de vértices diferentes. Pode-se observar isto em (11) onde obtém-se a assinatura comparável da imagem $\tilde{\xi}$ mod.

$$
\xi=\left(\lambda_{t, 0}, \lambda_{t, 1}, \ldots, \lambda_{t, 4}, \lambda_{t, 5}\right)
$$

$$
\begin{gathered}
\xi \bmod =\left(\left|\lambda_{t, 0}\right|,\left|\lambda_{t, 1}\right|, \ldots,\left|\lambda_{t, 4}\right|,\left|\lambda_{t, 5}\right|\right) \\
\tilde{\xi} \bmod =\frac{\xi \bmod }{\sum_{k=1}^{60} \xi \bmod _{k}}
\end{gathered}
$$

\subsection{Classificação Evolutiva}

A utilização de um classificador evolutivo é justificada pelo fato deste não necessitar de um conjunto de dados inicial, podendo, evoluir na quantidade de centros (grupos) sem a necessidade de uma parametrização prévia da quantidade de classes (Lughofer, 2011).

Além disso os algoritmos evolutivos oferecem a possibilidade de aprendizagem contínua, que as RNA (que são amplamente utilizadas) não oferecem. 
Agrupamento Evolutivo $\mathrm{O}$ algoritmo utilizado para o agrupamento se baseia nos algoritmos propostos por Lughofer $(2008,2011)$. Sua implementação foi realizada na linguagem Python versão 2.7 através do auxilio do pacote Numpy para computação científica, e seu funcionamento pode ser visto na figura 4 e através do Algoritmo 1.

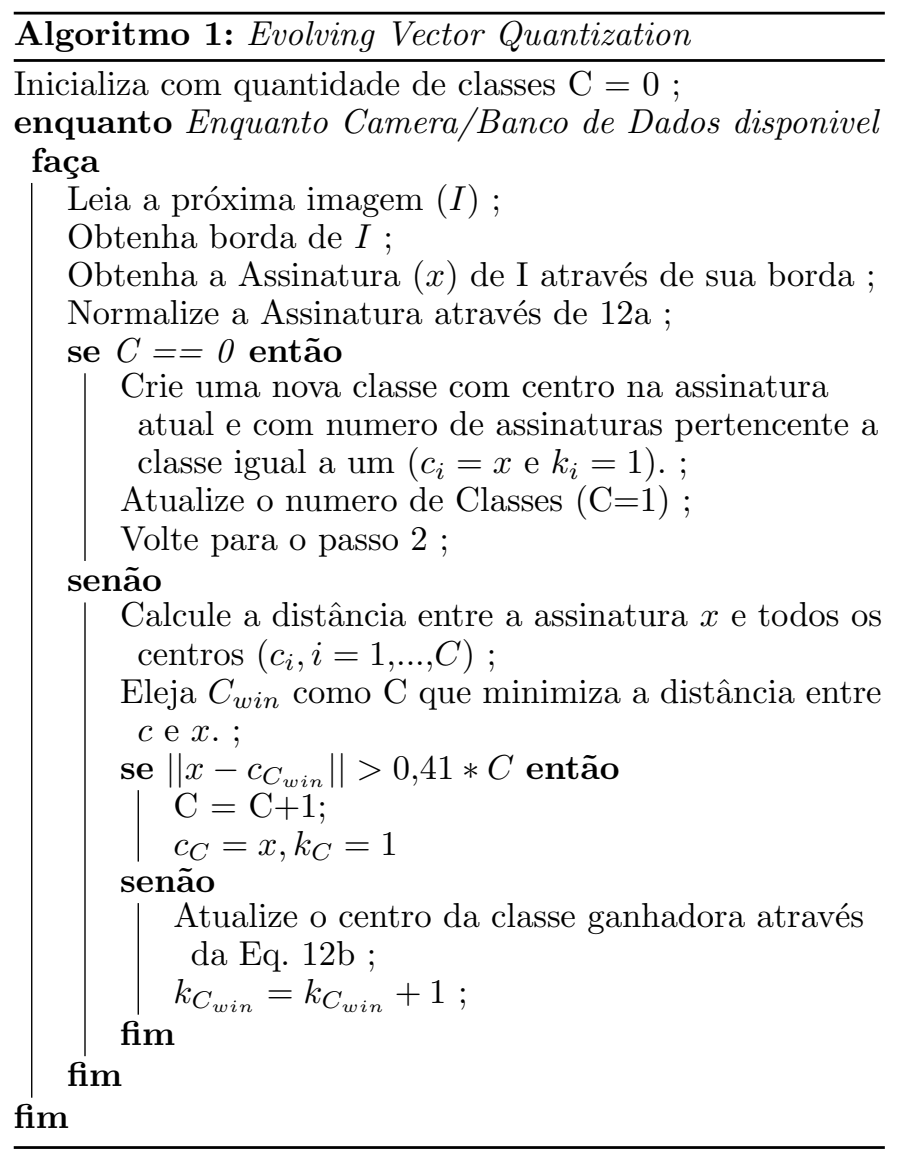

Entre suas principais características está "A habilidade de evolução dos centros de maneira incremental sem a necessidade de uma predefinição do número de centros"(Lughofer, 2011), característica essencial para utilização dessa metodologia de maneira on-line.

As equações (12a) e (12b) fazem parte da atualização do algoritmo e indicam, respectivamente, a normalização dos dados de entrada para uma faixa de valores entre 0 e 1 e a atualização do centro de uma classe $i$ dado a assinatura atual.

$$
\begin{gathered}
x=\frac{X-X_{\min }}{X_{\max }-X_{\min }} \\
c_{i}=c_{i}+\frac{0,5\left(x-c_{i}\right)}{k_{i}}
\end{gathered}
$$

A condição de criação de um novo cluster é definida como $d \geq 0,41 * C$, sendo $C$ a quantidade de Clusters e $d$ a distância euclidiana entre a assinatura e o cluster mais próximo. Essa condição faz com que o algoritmo tenda a atualizar seus centros em detrimento de criar novos.

\section{RESULTADOS}

Para a avaliação dos resultados foi realizado a identificação de bordas, obtenção da assinatura e agrupamento de um

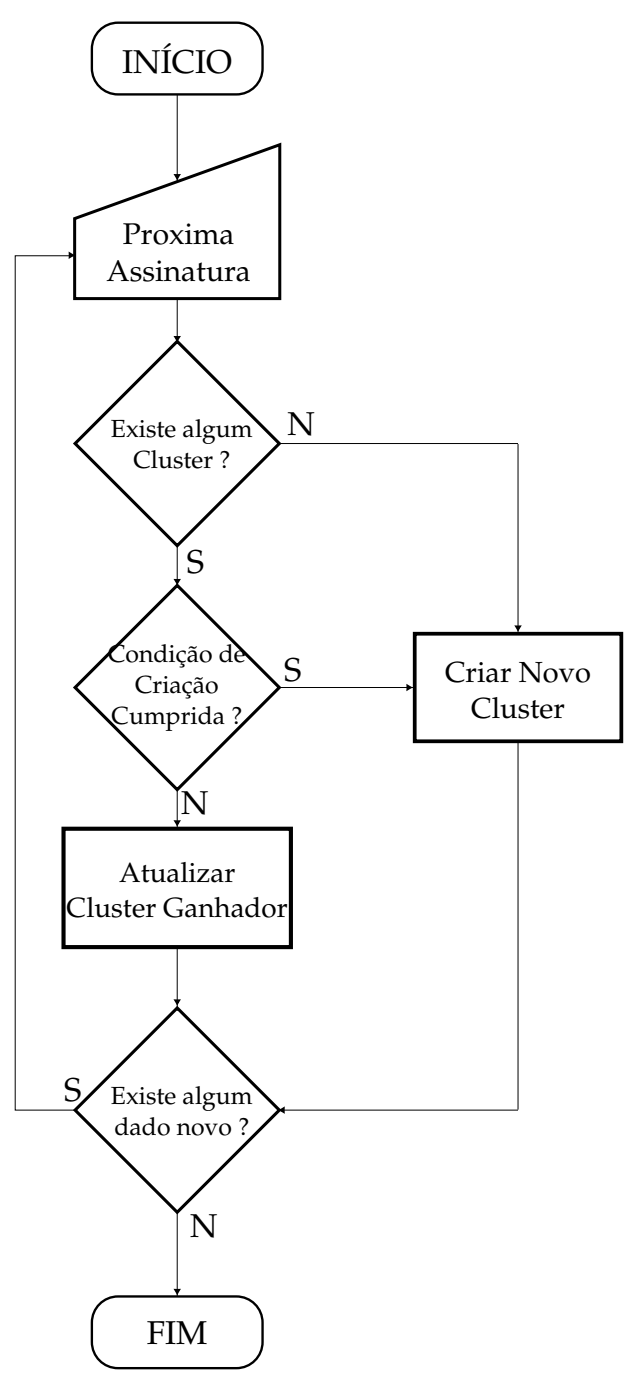

Figura 4. Fluxograma EVQ

subconjunto composto por 100 figuras, divididas em cinco classes da DataBase MPEG7, citada anteriormente, que foram ordenadas de maneira aleatória a fim de evitar influencia direta da ordenação das imagens nos resultados obtidos. Na figura 5 podem ser observados um exemplar de cada uma das classes.

O resultado do processamento das assinaturas pode ser visto na tabela 1 que mostra a quantidade de formas associadas a cada centro. Nota-se que o algoritmo criou 6 centros para as cinco classes sendo o centro 3 (C-3) o que recebeu menos elementos (apenas 4). Os agrupamentos C-1 recebeu 70\% das garrafas, C-2 e C-5 receberam, respectivamente, $95 \%$ das crianças e celulares. Enquanto C-4 e C-6 receberam, cada um, $100 \%$ dos carros e das Fontes.

Em comparação com as RNA normalmente utilizadas para a classificação, a principal vantagem da utilização do EVQ é o treinamento realizado de maneira online conforme os novos dados são inseridos. Uma característica notada na execução do código foi o reaproveitamento de C-2 que inicialmente foi criada para representar garrafas (assim como C-1) para a classificação de crianças. 


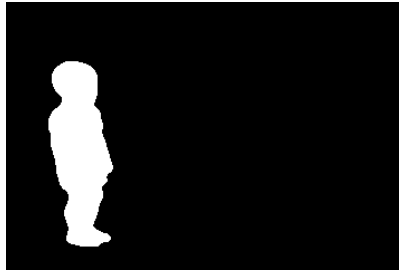

(a) Criança

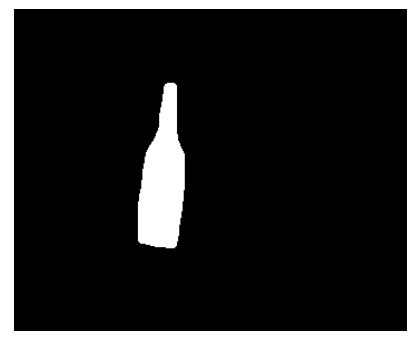

(c) Garrafa

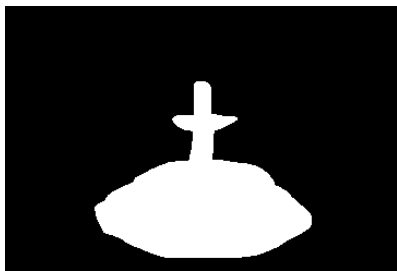

(b) Fonte

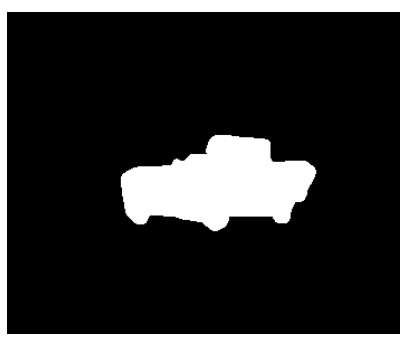

(d) Carro

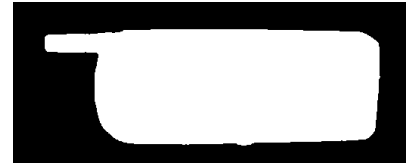

(e) Celular

Figura 5. Exemplos da Subclase MPEG7

\section{CONCLUSÃO}

O desenvolvimento da metologia proposta neste artigo propõe a utilização de técnicas clássicas de obtenção de bordas, em conjunto com abordagens modernas de descrição e classificação das mesmas. O sucesso na obtenção de uma classificação evolutiva, a partir da assinatura gerada pela análise da rede complexa associada a borda, sem a necessidade de um longo treinamento prévio mostra uma vantagem de utilização do método.

De maneira geral, o algoritmo se mostrou eficiente, com a velocidade de aprendizagem, e capaz de lidar com objetos que possuem bordas semelhantes, além de possuir a capacidade de adaptação dos centros a novas classes semelhantes.

O módulo de obtenção de bordas é capaz de lidar com diferentes tipos de objetos de forma a obter contornos condizentes com a imagem recebida. Embora, sejam necessárias alterações futuras para a inclusão do tratamento de sombras. A utilização do descritor de forma $2 \mathrm{D}$ proposta por Bof and Bof (2016) se mostrou eficiente para a geração de uma assinatura comparável, além de possuir poucos passos para a implementação. Como sugestão para projetos futuros e melhorias está a utilização de outras medidas de distância, tanto no descritor quanto no EVQ,

Tabela 1. Distribuição das figuras através dos Cluster

\begin{tabular}{cccccc}
\hline & Garrafa & Carro & Celular & Criança & Fonte \\
\hline C-1 & 14 & 0 & 1 & 1 & 0 \\
C-2 & 2 & 0 & 0 & 19 & 0 \\
C-3 & 4 & 0 & 0 & 0 & 0 \\
C-4 & 0 & 20 & 0 & 0 & 0 \\
C-5 & 0 & 0 & 19 & 0 & 0 \\
C-6 & 0 & 0 & 0 & 0 & 20 \\
\hline
\end{tabular}

e o aprimoramento da regra de criação de novos Cluster com a utilização de uma variância associada a cada centro.

\section{AGRADECIMENTOS}

Agradeço a Fundação de Amparo à Pesquisa e ao Desenvolvimento Científico e Tecnológico do Maranhão - FAPEMA e ao Instituto Federal de Educação, Ciência e Tecnologia - IFMA pelo apoio e suporte a pesquisa.

\section{REFERÊNCIAS}

Bicego, M., Murino, V., and Figueiredo, M.A. (2004). Similarity-based classification of sequences using hidden markov models. Pattern Recognition, 37(12), 2281-2291.

Bof, A. and Bof, P. (2016). Descritor de formas 2D baseado no espectro de redes complexas: Uma aplicação da teoria espectral de grafos e de redes complexas (Portuguese Edition). Novas Edições Acadêmicas.

Corke, P. (2017). Robotics, Vision and Control: Fundamental Algorithms In MATLABß Second, Completely Revised, volume 118. Springer.

Erpen, L.R.C. (2004). Reconhecimento de padrões em imagens por descritores de forma.

Gonzalez, R.C. (2010). Processamento Digital de Imagens. Pearson.

Gonzalez, R.C. and Woods, R.E. (2000). Processamento de imagens digitais. Edgard Blucher.

Henrique R. Z. da Silva, Guilherme de S. Weizenmann, R.M. (2018). Algoritmo para reconhecimento automatizado de defeitos em frangos de linha de produção utilizando visão computacional. In Congresso Brasileiro de Automática, 2018, 8. SBA.

Jha, A., Chiddarwar, S.S., and Andulkar, M. (2015). Application of human arm kinematics for robot path programming using imitation. In Proceedings of the 2015 Conference on Advances In Robotics, 15. ACM.

Lopes, A.K., Soares, F.A., Lopes, L.C., Laureano, G.T., Oliveira, L.L., Costa, R.M., and Soares, A.S. (2015). Identificação de postes da rede elétrica a partir de imagen do google street view. Anais do XII Simpósio Brasileiro de Automação Inteligente. Natal-RN: Sociedade Brasileira de Automática.

Lughofer, E. (2008). Extensions of vector quantization for incremental clustering. Pattern Recognition, 41(3), 9951011.

Lughofer, E. (2011). Evolving fuzzy systems-methodologies, advanced concepts and applications, volume 53. Springer.

Otsu, N. (1979). A threshold selection method from graylevel histograms. IEEE transactions on systems, man, and cybernetics, 9(1), 62-66.

Thiago S, F., Rodrigues, N, R., Júnior, M., Amaral, R., Asevedo, V., Rodruiges, N., Botelho, S., and Werhili, A. (2016). Sistema de visão de máquina para instrumentação de processos industriais. In Congresso Brasileiro de Automática, 2016, 6. SBA.

Zago, G.T., Dalvi, R.d.F., and Andreão, B.D.A. (2016). Metodologia computacional de processamento digital de imagens de fundo de olho para o diagnostico de glaucoma. In Congresso Brasileiro de Automática, 2016, 6. SBA. 